\title{
Texture size specificity in the slant aftereffect
}

\author{
ALLEN MILEWSKI \\ De Paul University, Chicago, Illinois 60614 \\ and \\ ALBERT YONAS \\ University of Minnesota, Minneapolis, Minnesota 55455
}

\begin{abstract}
Transfer of the median plane slant aftereffect was assessed across changes in stimulus texture size (sine-wave grating frequency). Under binocular viewing, reliable decrements in aftereffect magnitude were observed when texture size was changed, compared with no-change control conditions. Under monocular viewing conditions, no significant aftereffects were found. The results indicate a spatial-frequency-specific component of binocular slant aftereffects.
\end{abstract}

After a subject has inspected a surface slanted into depth for a prolonged period of time, a number of changes are observed in his judgment of slant. First, he judges the inspected surface to be less slanted and closer to the frontoparallel plane than it really is (Bergman \& Gibson, 1959). Moreover, he judges surfaces that are actually in the frontoparallel position to be slanted in the direction opposite the inspection slant (Kohler \& Emery, 1947). Finally, after slant inspection, a subject who is instructed to adjust the surface to the frontoparallel position shows an adjustment error in the direction of the inspection slant (Bergman \& Gibson, 1959; Wenderoth, 1970, 1971). These phenomena describe the "median plane slant aftereffect."

This aftereffect has been interpreted by Coltheart (1971) and others within a general model of feature analysis. Coltheart's interpretation proposes hypothetical, higher order slant analyzers. Each slant analyzer is assumed to respond selectively to a small range of visual three-dimensional slants and is also assumed to adapt under prolonged appropriate stimulation.

Proposals have differed with respect to the type of information that might be utilized by these slant analyzers. Coltheart has suggested one possibility involving binocular disparity detectors each sensitive to different amounts of disparity. If the receptive fields of a set of disparity detectors were arranged appropriately on the retina, they could provide in-

A preliminary version of this paper was presented at the April 1976 meetings of the Eastern Psychological Association. The research was completed while the first author was a postdoctoral fellow (NICHD No. 1F22 HD02723-01) at the Center for Research in Human Learning, University of Minnesota. The authors gratefully acknowledge the assistance of Daniel Zimmerman and Deborah Miller. Reprint requests should be sent to: A. E. Milewski, PhD, Department of Psychology, De Paul University, Chicago, Illinois 60614. formation about relative depth to a higher-order slant analyzer. An alternative proposal has been made by Wenderoth (1971) that involves differences in the two-dimensional tilt of contours received by each eye when viewing a slanted surface. The distinction is made here between tilt in two dimensions and slant into the third dimension. Wenderoth has compared the variations in magnitude of slant aftereffects across a range of inspection slants with variations in tilt aftereffects across a corresponding range of inspection tilts. From the reasonable similarity of these functions, Wenderoth has proposed that the slant aftereffect may reduce to a special case of the tilt aftereffect in which tilt aftereffects of opposite directions are simultaneously induced in the two eyes.

The implication of Wenderoth's proposal is that the slant aftereffect may be initially mediated by orientation detectors rather than by disparity detectors. Some neurophysiological evidence for the importance of contour orientation in slant perception has been provided by Blakemore, Fiorentini, and Maffei (1972). They report evidence in cats for binocularly driven cortical cells that respond maximally when contours presented separately to each eye differ slightly in orientation. On the other hand, a number of reports have shown slant aftereffects with randomly textured surfaces that provide no clear contours (Bergman \& Gibson, 1959; Robertson, Note 1). These studies question the proposal that slant aftereffects are based on contour orientation.

If the slant and tilt aftereffects are mediated by a common contour orientation mechanism as Wenderoth suggests, then one might expect that variables affecting the tilt aftereffect would also effect aftereffects of slant. One variable that appears to be important for the tilt aftereffect is stimulus grating size or spatial frequency. While the literature is not unanimous (see Campbell \& Maffei, 1971; 
Parker, 1972), recent careful analyses by Sullivan (cited in Sekuler, 1974) and by Ware and Mitchell (1974) have demonstrated clear evidence for spatial frequency specificity in the tilt aftereffect. In these studies, tilt aftereffects were either eliminated or reduced in magnitude in conditions where the spatial frequency of the stimulus was changed between adaptation and test. A closely related study on the tilt illusion has also found evidence of spatial frequency specificity (Georgeson, 1973).

In contrast to this evidence indicating size specificity in the tilt aftereffect, there is no existing data on a similar specificity in the slant aftereffect. If no size specificity was found for the slant aftereffect, a contour orientation mechanism for the slant aftereffect such as Wenderoth proposes would be put into question. The present study therefore investigated transfer of slant aftereffects across a change in surface texture size.

\section{EXPERIMENT 1}

\section{Method}

Subjects. The subjects were 12 undergraduate college students.

Apparatus. Vertical sine-wave gratings were produced on the face of a slanting oscilloscope. Two texture sizes were employed: the small texture was a grating of $3.7 \mathrm{cycles} / \mathrm{deg}$, and the large texture was a grating of $1.2 \mathrm{cycles} / \mathrm{deg}$. Contrast was approximately 0.6 .

The stimulus was viewed in a darkened room through a tube that was parallel to the oscilloscope face. A single surface mirror slanted at $45 \mathrm{deg}$ toward the stimulus was interposed at the end of the tube, resulting in a straight view of a continuous grating subtending $8.6 \mathrm{deg}$. Subjects viewed the stimulus from a distance of $46 \mathrm{~cm}$. A manually operated shutter oceluded the stimulus between inspections and tests. Gross head movements were reduced by a headrest.

An adjustment procedure was used in which the subject was instructed to set the stimulus surface to the frontoparallel position. The subject could manually slant the stimulus surface through the median plane by turning a wheel with his left hand. Slant measurement was made relative to gravitational upright and was accurate to $0.5 \mathrm{deg}$. In this experiment, a surface slanted with the top toward the subject was denoted positive degrees slant; slant with the surface top away from the subject was recorded as negative degrees slant.

Procedure. Following from four to six practice adjustments, each subject was tested in three conditions; (1) in the size-change condition, subjects initially inspected one texture size slanted at either positive or negative $25 \mathrm{deg}$ from gravitational upright and made a test adjustment with the other texture size; (2) in the size-same condition, subjects initially inspected one size texture at either positive or negative $25 \mathrm{deg}$ and made a test adjustment with the same size texture; (3) in the control condition, subjects inspected one size texture set at the frontparallel position and made a test adjustment with the same size texture. Each condition consisted of two trials, one with the small texture test stimulus and the other with the large texture test stimulus. Four successive test adjustments were made on each trial. The first adjustment followed $90 \mathrm{sec}$ of adaptation; the remaining three followed $30 \mathrm{sec}$ of adaptation. The interval between each inspection and test was approximately $10 \mathrm{sec}$, during which a shutter blocked the subject's view. Within a trial, the four adjustments were counterbalanced for the starting position direction, which was either a positive or negative 25 -deg slant. Since half the subjects received initial inspection slants in the positive direction and half received negative inspection slants, the design, across subjects, eliminated confounding effects of starting position which have previously been reported for adjustment studies of aftereffects (Wenderoth, Rodger, \& Curthoys, 1968). The subjects were instructed to make adjustments within $10 \mathrm{sec}$. To reduce the effects of response learning, the adjustment wheel's gear ratio was randomly switched to fast or slow on each trial.

\section{Results}

In order to provide a sensitive measure of aftereffect magnitude in experimental conditions relative to control conditions, difference scores were computed for each subject by subtracting the mean frontoparallel adjustment setting in the four appropriate control trials from the mean adjustment in each of the experimental conditions. A mixed-design analysis of variance indicated a reliable difference between size-change and size-same conditions $[F(1,11)=9.86, p<.01]$. Since no main effects or interactions of stimulus size were found, data were collapsed across stimulus size, and the mean difference scores for the size-change and size-same conditions are shown in Figure 1.

Nine of 12 subjects in this experiment showed less aftereffect in both size-change conditions compared with the size-same conditions; for the remaining three subjects, one size-same condition always showed more aftereffect than its comparable sizechange condition. In order to determine whether anyy tíansfer of aftereffect occurred across a size change, a comparison was made of the mean difference scores for the combined size-change conditions with zero. A one-tailed $t$ test failed to show significant transfer $[t(11)=.075]$.

The differences found between size-change and size-same conditions, in addition to the failure to find significant aftereffects in the size-change condi-

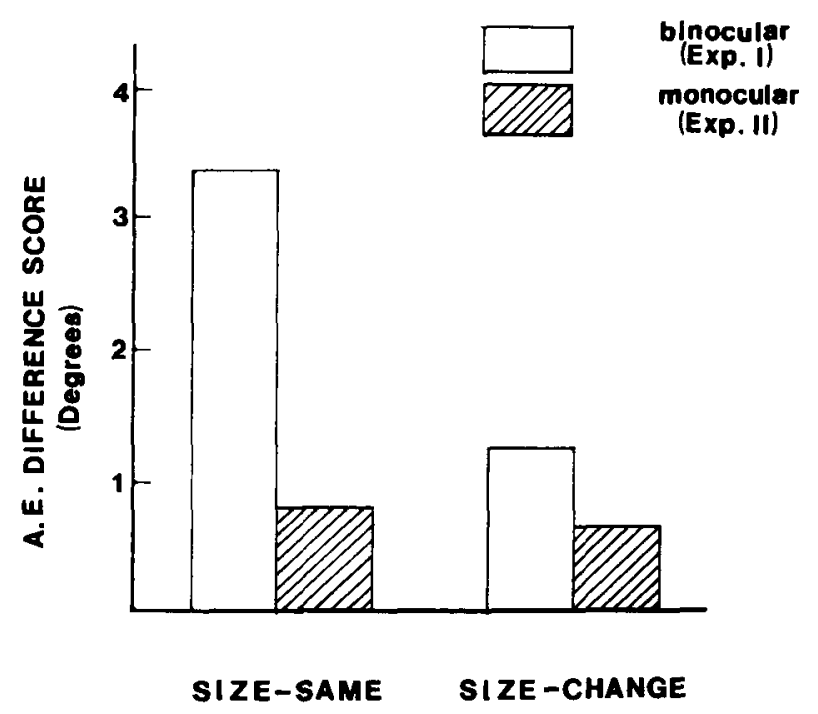

Figure 1. Mean aftereffect difference scores for size-same and size-change conditions under binocular viewing of Experiment 1 (solid) and monocular viewing of Experiment 2 (hatched). 
tion, indicate a large slant aftereffect component that does not transfer across changes in texture size. This result, then, is analogous to those indicating spatial frequency specificity in the two-dimensional tilt aftereffect.

However, it may be possible to explain the result of this experiment by assuming that subjects were adjusting not on the basis of slant at all, but rather solely on the basis of monocular tilt of the stimulus grating. Subjects had been instructed to view with both eyes, and also to attend to slant and not to two-dimensional tilt, but it was decided to test this possibility in a second experiment which replicated the conditions of Experiment 1 under monocular viewing conditions. If monocular viewing conditions produce minimal aftereffect compared with those found with binocular viewing, it can be argued that the results of Experiment 1 were mediated largely by binocular depth information.

\section{EXPERIMENT 2}

\section{Method}

Procedure. The conditions of Experiment 2 were identical to those of Experiment 1 with the exception that stimuli were viewed monocularly with the subject's dominant eye. The subjects were again instructed to adjust on the basis of slant.

\section{Results}

The results are shown by the hatched bars in Figure 1. An analysis of variance on difference scores for Experiment 2 indicated no significant main effects or interactions of experimental condition or test stimulus size. A one-tailed $t$ test comparing mean difference scores for all experimental groups with zero failed to indicate a reliable aftereffect in any of the conditions $[t(11)=0.68]$. The subjects appeared to have considerable difficulty adjusting slant monocularly; the adjustment variance was high and considerably greater than under the binocular conditions of Experiment 1.

\section{DISCUSSION}

The results of Experiment 1 provide evidence of a texture-size-specific component in the median plane slant aftereffect, by showing a significant difference in aftereffect magnitude between size-same and sizechange conditions and by failing to demonstrate a reliable aftereffect in the size-change condition. It is possible to view the present result in terms of a general feature analyzer model similar to that proposed by Coltheart (1971) but with the additional property that units selectively respond and adapt not only to specific slants but also to a specific range of spatial frequencies. It should be noted that the present study can properly draw this conclusion only for conditions involving binocular depth information, since the failure of Experiment 2 to show reliable monocular aftereffects suggests that monocular cues were minimal. While monocular aftereffects have been reported elsewhere with highly textured surfaces (Bergman \& Gibson, 1959), the present study provides no information about spatial frequency specificity in monocular situations.

The spatial frequency specificity found in this study of the slant aftereffect is similar to that reported in previous literature for the twodimensional tilt aftereffect. This study therefore does not rule out Wenderoth's (1971) proposal that both slant and tilt aftereffects are mediated by a common contour orientation mechanism. On the other hand, the present findings obviously do not establish with certainty Wenderoth's proposal. It is possible for example that the two aftereffects are mediated by separate mechanisms, both showing spatial frequency specificity. Regardless of the mechanism involved in the slant aftereffect, these data are consistent with a growing number of findings (Sekuler, 1974) in suggesting that spatial frequency specificity is a pervasive aspect of visual functioning.

\section{REFERENCE NOTE}

1. Robertson, L. S. Adaptation to a visual gradient of texture density. Paper presented at meetings of the Midwestern Psychological Association. Chicago. 1976.

\section{REFERENCES}

Bergman. R..\& Gibson, J. J. The negative after-effect of the perception of a surface slanted in the third dimension. American Journal of Psychology, 1959, 72, 363-374.

Blakemore, C., Fiorentini, A., \& Maffei, L. A second neural mechanism of binocular depth discrimination. Journal of Physiology, 1972, 226. 725-749.

Campbell, F. W.. \& Maffei, L. The tilt aftereffect: A fresh look. Vision Research, 1971, 11. 833-840.

Coltheart, M. Visual feature-analysis and aftereffects of tilt and curvature. Psychological Review, 1971, 78, 114-121.

Georgeson, M. A. Spatial frequency specificity of a visual tilt illusion. Nature, 1973, 245, 43-45.

Kohler. W.. \& Emery, D. Figural after-effects in the third dimension of visual space. American Journal of Psychology, 1947. 60. 159-201.

Parker, D. M. Contrast and size variables and tilt aftereffect. Quarterly Journal of Experimental Psychology, 1972, 24, 1-7.

SeKuler, R. Spatial vision. Annual Review of Psychology, 1974, 25. 195-231.

W ARE, C., \& MrTChell, D. E. The spatial selectivity of the tilt after-effect. Vision Research, 1974, 14, 735-737.

WENDEROTh, P. M. A visual spatial aftereffect of surface slant. American Journal of Psychology, 1970, 83, 576-590.

WENDERoth, P. M. Studies of a stereoscopic aftereffect of a contour slanted in the median plane. Australian Journal of Optometry, April 1971, 114-123.

Wenderoth, P. M., Rodger, R. S., \& Curthoys, I. S. Confounding of psychophysical errors and sensory effects in adjustment measures of spatial aftereffects. Perception \& Psychophysics, 1968, 4. 133-138.

(Received for publication June 28, 1976; revision received September 24, 1976.) 